\title{
Translation and validation of Training Needs Analysis Questionnaire among reproductive, maternal and newborn health workers in Tanzania
}

Tumbwene Mwansisya ${ }^{1 *}$, Columba Mbekenga ${ }^{1}$, Kahabi Isangula ${ }^{1}$, Loveluck Mwasha ${ }^{1}$, Eunice Pallangyo ${ }^{1}$, Grace Edwards ${ }^{2}$, James Orwa ${ }^{3}$, Michaela Mantel ${ }^{3}$, Micheal Mugerwa ${ }^{4}$, Leonard Subi ${ }^{5}$, Secilia Mrema ${ }^{6}$, David Siso ${ }^{7}$, Edna Selestine ${ }^{4}$ and Marleen Temmerman ${ }^{3}$

\begin{abstract}
Background: Continuous professional development (CPD) has been reported to enhance healthcare workers' knowledge and skills, improve retention and recruitment, improve the quality of patient care, and reduce patient mortality. Therefore, validated training needs assessment tools are important to facilitate the design of effective CPD programs.

Methods: A cross-sectional survey was conducted using self-administered questionnaires. Participants were healthcare workers in reproductive, maternal, and neonatal health (RMNH) from seven hospitals, 12 health centers, and 17 dispensaries in eight districts of Mwanza Region, Tanzania. The training needs analysis (TNA) tool that was used for data collection was adapted and translated into Kiswahili from English version of the Hennessy-Hicks' Training Need Analysis Questionnaire (TNAQ).

Results: In total, 153 healthcare workers participated in this study. Most participants were female $83 \%(n=127)$, and $76 \%(n=115)$ were nurses. The average age was 39 years, and the mean duration working in RMNH was 7.9 years. The reliability of the adapted TNAQ was 0.954 . Assessment of construct validity indicated that the comparative fit index was equal to 1.
\end{abstract}

Conclusions: The adapted TNAQ appears to be reliable and valid for identifying professional training needs among healthcare workers in RMNH settings in Mwanza Region, Tanzania. Further studies with larger sample sizes are needed to test the use of the TNAQ in broader healthcare systems and settings.

Keywords: Validation, Training need analysis, Reproductive, Maternal, Newborn, Healthcare workers

\footnotetext{
* Correspondence: tumbwene.mwansisya@aku.edu

${ }^{1}$ School of Nursing and Midwifery, the Aga Khan University, Dar es Salaam, Tanzania

Full list of author information is available at the end of the article
}

(c) The Author(s). 2021 Open Access This article is licensed under a Creative Commons Attribution 4.0 International License, which permits use, sharing, adaptation, distribution and reproduction in any medium or format, as long as you give appropriate credit to the original author(s) and the source, provide a link to the Creative Commons licence, and indicate if changes were made. The images or other third party material in this article are included in the article's Creative Commons licence, unless indicated otherwise in a credit line to the material. If material is not included in the article's Creative Commons licence and your intended use is not permitted by statutory regulation or exceeds the permitted use, you will need to obtain permission directly from the copyright holder. To view a copy of this licence, visit http://creativecommons.org/licenses/by/4.0/. The Creative Commons Public Domain Dedication waiver (http://creativecommons.org/publicdomain/zero/1.0/) applies to the data made available in this article, unless otherwise stated in a credit line to the data. 


\section{Background}

Reproductive, maternal, newborn, child, and adolescent healthcare remains a challenge in many low-income countries. For example, Tanzania failed to meet the Millennium Development Goal (MDG) target of reducing the maternal mortality ratio (MMR) to 228 per 100,000 live births by 2015 [1]. The MMR in Tanzania remains unacceptably high, despite the slight decline between the 1990 s (578 per 100,000 live births) and 2016 (556 per 100,000 live births) [1, 2]. In addition, the national neonatal mortality rate (NMR) remains high, at 25 deaths per 1,000 live births [2].

Although there are many drivers of poor maternal and newborn health, low quality of reproductive healthcare services was identified as an important contributor [3]. The poor quality of delivery services may be associated with shortage of skilled birth attendants [4]. Previous studies estimated that $20-21 \%$ of neonatal deaths could be prevented by healthcare workers' promotion of simple, evidence-based practices (e.g., exclusive breastfeeding and hand washing) and prevention of hypothermia and infection $[5,6]$. Other studies indicated that healthcare workers were inadequately supported in terms of training and remuneration, lack of skills mix, and poor retention strategies [7]. Moreover, the Tanzania Government budget allocation to reproductive, maternal, and neonatal health (RMNH) is limited and inconsistent [3]. Taken together, these factors lead to inadequate quality of healthcare systems.

Continuous professional development (CPD) may help address this issue, as it has been reported to enhance healthcare workers' knowledge and skills, improve retention and recruitment, improve the quality of patient care, and reduce patient mortality [8, 9]. In addition, skilled birth attendants play a vital role in ensuring optimal RMNH practices by communicating with care and respect and having the requisite knowledge and technical proficiency [5]. It is therefore important to assess the training needs of RMNH staff to facilitate the development of capacity building interventions to bridge existing skill-related gaps, thereby improving the quality of healthcare systems.

An important tool in identifying healthcare workers' knowledge and skills gaps and establishing future CPD training profiles is training needs analysis (TNA) [8]. Context-specific TNA can facilitate accurate assessment of healthcare workers' training needs for successful RMNH programs [10]. Furthermore, a validated TNA tool to assess training needs in low-income countries is needed to identify the context-specific underdeveloped skills, insufficient knowledge, or inappropriate attitudes among healthcare workers. Despite the contribution of CPD in reducing the MMR and NMR, research shows that TNA in many organizational settings has been unsystematic or used tools that were not validated [11]. This highlights the need for validated TNA tools to facilitate the design of effective CPD programs.

The Aga Khan Development Network is working with the Tanzania Ministry of Health, Community Development, Gender, Elderly and Children to improve access to RMNH in Mwanza Region, Tanzania (IMPACT project). This 4-year project (2017-2021) focused on reproductive health services, and aimed to accelerate the reduction of maternal and newborn mortality in Tanzania by addressing major RMNH challenges in eight districts of Mwanza Region. The IMPACT project combines training with mentorship interventions that have been reported to be important strategies to support skills and capacity transfer among in-service healthcare workers [12], which in turn improves RMNH outcomes [13]. In the project's baseline survey, we conducted TNA among healthcare workers in RMNH in Mwanza Region. The IMPACT project team developed a TNA tool based on adapting items from the Hennessy-Hicks Training Needs Analysis Questionnaire (TNAQ) [14], which was developed in 1996 to evaluate training needs and priorities of healthcare professionals. The TNAQ has been used in both developing and developed countries [1416]. This study aimed to validate the adapted TNAQ in the local Tanzanian context, with the goal of facilitating development of effective needs-based CPD programs to improve healthcare workers' competence in the delivery of RMNH and child and adolescent healthcare services in Mwanza Region.

\section{Materials and methods \\ Study Area}

Details of the study settings have been reported elsewhere [17]. Briefly, this study was conducted in Mwanza Region, which is in the northern part of Tanzania bordering Lake Victoria. The 2012 national census indicated Mwanza Region had a population of $2,772,509$ people in an area of $35,187 \mathrm{~km}^{2}$ [2]. The average annual population growth rate for 2002-2012 was 3.0\%, making Mwanza among eight regions in Tanzania with a high growth rate. The Tanzania Human Development Report ranked Mwanza Region as 13th among Tanzania's 35 regions, with one-third of the population living in severe poverty $(32.8 \%)$ and one-fifth of the population vulnerable to poverty $(19.7 \%)$ compared with national averages of 31.3 and $18.2 \%$, respectively [1].

The region is part of the Lake Zone, where the MMR was 453 deaths per 100,000 live births and the underfive mortality rate was 88 deaths per 1,000 live births in the 10-year period preceding the 2015/16 Tanzania Demographic and Health Survey; these rates failed to meet Tanzania's MDG targets [2]. Available data on the NMR of Mwanza Region in 2015 showed there were 29 
deaths per 1,000 live births, which was higher than the national average of $25 / 1,000$ [2].

National data indicate that only $50.7 \%$ of pregnant women attended at least the four recommended health facility visits for focused antenatal care during their last pregnancy [2]. Health facility deliveries in Mwanza Region account for $63.6 \%$ of deliveries on average, although there are persisting large disparities, with $87 \%$ of deliveries occurring in facilities in urban areas versus $54.7 \%$ in rural areas [2]. Given the poor RMNH indicators, Mwanza Region is one of five regions in Tanzania that have been prioritized by the Government [2]. Understanding the training needs of healthcare workers in Mwanza Region formed an important entry point for the IMPACT project in seeking to improve RMNH indicators. This provided the rationale for validating the TNAQ in this region.

\section{Design}

We conducted a cross-sectional survey using selfadministered questionnaires. The survey involved healthcare workers in RMNH at selected health facilities in all eight districts of Mwanza Region, Tanzania.

\section{Study population}

All adult healthcare workers responsible for RMNH service provision that were present in the selected facilities at the time of this survey were eligible to participate.

\section{Eligibility and sampling}

Eligible participants were healthcare workers responsible for RMNH services. The selected healthcare workers were from 36 stratified health facilities randomly sampled from the 80 IMPACT project-supported sites. These healthcare facilities comprised seven district hospitals, 12 health centers, and 17 dispensaries. All healthcare workers providing RMNH services who were available at the time of the study visit were included. In total, 153 healthcare workers were used to validate the TNAQ, giving an item to participant ratio of 1:3 [18]. This ratio related to the finite sample of healthcare workers in RMNH in the selected facilities. Although some studies suggested an item to participant ratio of 1 : 10 was appropriate, other studies used ratios of 1:2 [18]. Therefore, the sample size used in this study was considered adequate.

\section{Data collection tool}

The TNAQ was designed for RMNH providers at the primary (dispensary and health center) and secondary (health center and district and designated district hospital) levels. As noted above, the tool was adapted from that developed by Hennessy-Hicks [14, 19], which has been psychometrically tested for reliability and validity and adopted by the World Health Organization (WHO) [19]. Several other TNA questionnaires were considered, including the Professional Nurse Self-Assessment Scale of Clinical Core Competencies [20], Addiction Medicine Training Needs Analysis Scale [21], and Community Competence-based Questionnaire [22]. However, the Hennessy-Hicks TNAQ was selected because it was developed specifically to evaluate healthcare professionals' training requirements and facilitate subsequent use of the findings to prioritize and meet local training needs [19]. The Hennessy-Hicks questionnaire measures a range of skills including clinical, managerial, administrative, and research audit activities. Responses to the TNAQ are on a seven-point Likert scale: "not at all important" (1), "slightly important" (2), "quite important" (3), "moderately important" (4), "important" (5), "very important" (6), and "extremely important" (7). Currently, there is no validated and standardized tool for assessing training needs in RMNH in Tanzania. There is also a shortage of skilled birth attendants in the region, meaning that the few who are available must perform a range of activities. Therefore, validation of the Hennessy-Hicks TNAQ was deemed necessary to capture the multiple responsibilities of RMNH personnel.

\section{Translation}

Two bilingual health professionals experienced in reproductive health and research provided a forward translation of the TNAQ to the Kiswahili language. This was followed by back translation by a bilingual non-health professional. The two versions were compared, and suggestions for modification incorporated in the Kiswahili version. The adapted TNAQ was then pilot tested with 12 healthcare professionals working in reproductive health and management. The feedback received from the pilot study was incorporated in the final adapted TNAQ. Minimum translation criteria were applied [23].

\section{Data collection procedure}

Data were collected in August 2017. During data collection, the person in-charge of each selected facility identified RMNH personnel for participation. The paper-based TNAQ was self-administered, and responses were confidential. The Questionnaires were administered to participants during their working hours to maximize their participation. Participants were requested to assess their own performance and rate the perceived importance of specific RMNH services/activities. Specifically, the questionnaire asked participants how important each RNMHrelated activity was to the successful performance of their work and how good they considered their performance in each activity. Participants were also asked to identify areas in which they most wanted to receive additional training, and to note the training that they had most recently 
completed. Research assistants were available to answer questions and clarify elements of the questionnaire as needed. Returned questionnaires were checked for completeness and accuracy before the research team left each health facility.

\section{Data management}

SPSS version 20.0 was used for data entry and statistical analyses. Data from the questionnaires were reviewed to identify consistencies and differences, coded, and quantified. The data were then manually entered into a password-protected database via an entry screen that performed validation checks for accuracy. Missing data were excluded during analysis. Analysis of Moment Structures (AMOS) software embedded in SPSS version 20 was used to confirm the factor structure of the TNAQ using an exploratory analysis.

\section{Data analysis and interpretation}

The adapted TNAQ was evaluated for both reliability and validity. Reliability was used to determine the stability and equivalence of sets of TNAQ items. Although reliability is necessary, it is not sufficient to determine the validity of an instrument [21]. Therefore, we also assessed the validity of the TNAQ to determine the degree to which the instrument could measure what it was is supposed to measure.

\section{Reliability of the scale}

The internal consistency reliability was determined by Cronbach's alpha. A Cronbach's alpha of 0.7 is considered to indicate acceptable reliability [21]. Moreover, the individual quality effect of each item was assessed using Cronbach's alpha to test the reliability if an item was deleted. Split-half reliability was calculated by randomly splitting the items into two sets to determine the consistency of the TNAQ across sub-groups. A Guttman split-half coefficient of $\geq 0.80$ indicates good internal consistency [21]. Finally, inter-rater reliability was used to evaluate the performance of the measure across different raters, and was determined by the intra-class correlation coefficient [24]; a coefficient $\geq 0.7$ indicates an acceptable level of inter-rater reliability.

\section{Validity of the scale}

\section{Face and content validity}

The Hennessy-Hicks instrument has been adapted to assess the training needs of different health care practitioners in a range of cultural contexts $[14,19,25]$. However, it has not previously been validated in the Tanzanian context where the culture may differ from countries where the instrument has been used. In adapting the TNAQ for this study, pooled items were obtained from a literature review and the opinion of experts experienced in the reproductive health field. The pooled items were validated by three experts with expertise in teaching, reproductive health, research, and the local culture including customs, traditions, and the local language (Kiswahili). The TNA tool divides the items into broad categories, allowing for both intracategory and inter-category comparison of training needs.

\section{Criterion validity}

We assessed concurrent validity, which is one of three types of criterion validity (predictive validity, concurrent validity, and postdictive validity) [26]. The TNAQ was found to have acceptable concurrent validity.

\section{Construct validity}

The sampling adequacy was determined by the KaiserMeyer-Olkin (KMO) measure and the suitability of factorization was assessed using Bartlett's test of sphericity [27]. Construct validity was evaluated using exploratory factor analysis (EFA) and confirmatory factor analysis (CFA). The factor structure was first evaluated with EFA, which was conducted using principal axis factoring estimation with promax rotation. This determines the underlying factor structure of the items, and has the advantages of being fast and conceptually simple [28]. The criteria applied for factor retention were: (a) eigenvalues greater than 1.0, (b) the percentage of total variance explained, (c) scree plot, and (d) factors with loadings above 0.40 . Items with a loading below 0.4 and those with cross loading over 0.4 were deleted. CFA was used to validate the factor structure obtained from the EFA. The CFA was determined by the factor loading > 0.4 by using the AMOS statistical program. The CFA indicated the adequacy of the data in the model and the appropriate fit of the structural model for the healthcare workers who are the study population. The variance and covariance matrix of the 49 items was determined by maximum likelihood minimization function and the 3 -factors model was assumed basing on our analysis. To adjust the scaling of the factors to that of the indicators, a fixed 1.0 factor loading and a fixed measurement error variance was applied. Model fit was considered acceptable if $\mathrm{X}^{2} / \mathrm{df}<2$, comparative fit index $(\mathrm{CFI})>0.9$, root mean square error of approximation $($ RMSEA) $<0.04[27,29]$.

\section{Convergent and discriminant validity}

Convergent Validity Convergent validity refers to how variables on a single factor are correlated [30]. We assessed the convergent validity of the adapted TNAQ with the average variance extracted (AVE) and composite reliability (CR). The AVE and CR were calculated manually using the formula suggested by Hair 
et al., [31]. An AVE $>0.5$ and $C R>0.7$ indicate convergent validity. Moreover, convergent validity was confirmed by the factor loadings. The significance of factor loading is based on the sample size. Generally, the smaller the sample size, the higher the factor loadings [18]. For example, a sample of 50 participants may require a sufficient factor loading of 0.75 whereas a sample of 350 may require a sufficient loading of 0.3 . Therefore, the sample size of 153 participants used in this study required factor loadings of 0.4 to be sufficient.

Discriminant validity To establish discriminant validity, the maximum shared variance (MSV) and average shared variance (ASV) were calculated, and their values compared. A MSV value less than the ASV value indicates discriminant validity [30]. Furthermore, the correlation matrix was examined with correlations between factors $<0.7$ indicating discriminant validity.

\section{Results}

Participants were 153 healthcare workers. Most participants were female $(8.73 \%, n=128)$ and the average age was 39 years. Nurses formed the largest group of participants $(76 \%, n=115)$, and the mean time in RMNH was 7.9 years. Moreover, most participants indicated they had experience of $0-10$ years in both general healthcare services and RMNH. Participants' sociodemographic details are presented in Table 1.

\section{Reliability of the adapted TNAQ}

The Cronbach's alpha of the TNAQ was 0.954, showing internal consistency. Moreover, deletion of each item individually resulted in a Cronbach's alpha $\geq 0.953$. The splithalf reliability was also good, with a Guttman split-half coefficient of 0.84 . The intra-class correlation coefficient was 0.954 showing inter-rater reliability. Table 2 presents details of the internal consistency for individual items.

\section{Content validity}

Pooled items that were obtained from the literature review and expert opinion were validated by an expert panel with expertise in teaching, reproductive health, research, and local culture including customs, traditions, and the local language (Kiswahili) that is spoken by $95 \%$ of Tanzanians. All items were found to be adequate and clear during translation (expert opinion) and pilot testing. This indicated good content validity. In terms of face validity, the adapted TNAQ had variables in a single factor that were related and easy to label. We found three factors: general reproductive health, intraoperative care, and comprehensive emergency obstetric and newborn care (CEmONC). These labels were related to the similar focus of the items in a single factor. Assessment of the criterion validity showed the adapted TNAQ had acceptable validity and reliability, similar to the original Hennessy-Hicks instrument. In terms of convergent validity, the AVE was 0.5 and the CR was 0.6. Moreover, all items had factor loadings above 0.4. The three factors each included variables that were highly inter-correlated

Table 1 Demographic characteristics and clinical experience of study participants working in reproductive health in Mwanza ( $n=153$ )

\begin{tabular}{|c|c|c|c|c|}
\hline Characteristic & $\begin{array}{l}\text { Dispensary } \\
(n=29)\end{array}$ & $\begin{array}{l}\text { Health center } \\
(n=53)\end{array}$ & $\begin{array}{l}\text { Hospital } \\
(n=70)\end{array}$ & $\begin{array}{l}\text { Total } \\
(n=152)^{\mathrm{a}}\end{array}$ \\
\hline Mean age (years) & 37.4 & 38.0 & 39.9 & 38.8 \\
\hline $\operatorname{Sex}(M, F)$ & 8,21 & 8,45 & 9,61 & 25,128 \\
\hline Mean years in service & 12.9 & 13.4 & 14.0 & 13.59 \\
\hline Mean years in $\mathrm{RMNH}$ & 8.1 & 10.1 & 6.1 & 7.9 \\
\hline \multicolumn{5}{|l|}{ Cadre } \\
\hline Assistant medical officers & $1(3 \%)$ & $1(2 \%)$ & $1(1 \%)$ & $3(2 \%)$ \\
\hline Clinical officers & $0(0 \%)$ & $2(4 \%)$ & $6(9 \%)$ & $8(5 \%)$ \\
\hline Enrolled nurses & $8(28 \%)$ & $22(42 \%)$ & $23(31 \%)$ & $53(35 \%)$ \\
\hline Registered nurses & $12(41 \%)$ & $22(42 \%)$ & $10(14 \%)$ & $62(41 \%)$ \\
\hline Medical attendant & $8(28 \%)$ & $6(11 \%)$ & $12(17 \%)$ & $26(17 \%)$ \\
\hline $\begin{array}{l}\text { Clinical experience } \\
\text { (years) }\end{array}$ & $\begin{array}{l}\text { In service } \\
\text { n (\%) }\end{array}$ & $\begin{array}{l}\text { In RMNH } \\
\text { n (\%) }\end{array}$ & & \\
\hline $0-10$ & $90(58.8)$ & $80(80.4)$ & & \\
\hline $11-20$ & $18(11.8)$ & $11(7.2)$ & & \\
\hline $21-30$ & $24(15.7)$ & $13(8.5)$ & & \\
\hline $30+$ & $19(12.4)$ & $7(3.9)$ & & \\
\hline
\end{tabular}

One participant had missing demographic data and was excluded from this analysis 
Table 2 Internal consistency of the items in the adapted Training Needs Analysis Questionnaire

\begin{tabular}{|c|c|c|c|c|}
\hline Item number & $\begin{array}{l}\text { Scale mean if } \\
\text { item deleted }\end{array}$ & $\begin{array}{l}\text { Scale variance if } \\
\text { item deleted }\end{array}$ & $\begin{array}{l}\text { Corrected item-total } \\
\text { correlation }\end{array}$ & $\begin{array}{l}\text { Cronbach's alpha } \\
\text { if item deleted }\end{array}$ \\
\hline 1. & 284.75 & 998.294 & 0.513 & 0.954 \\
\hline 2. & 284.81 & 1005.931 & 0.443 & 0.954 \\
\hline 3. & 284.74 & 993.973 & 0.551 & 0.954 \\
\hline 4. & 284.85 & 980.934 & 0.555 & 0.953 \\
\hline 5. & 284.53 & 998.081 & 0.594 & 0.954 \\
\hline 6. & 284.64 & 1000.593 & 0.460 & 0.954 \\
\hline 7. & 284.47 & 994.166 & 0.514 & 0.954 \\
\hline 8. & 284.40 & 1002.037 & 0.537 & 0.954 \\
\hline 9. & 284.86 & 990.278 & 0.445 & 0.954 \\
\hline 10. & 284.67 & 986.753 & 0.501 & 0.954 \\
\hline 11. & 284.44 & 991.086 & 0.588 & 0.953 \\
\hline 12. & 284.56 & 987.599 & 0.504 & 0.954 \\
\hline 13. & 284.95 & 974.630 & 0.464 & 0.954 \\
\hline 14. & 284.45 & 983.395 & 0.653 & 0.953 \\
\hline 15. & 284.42 & 990.877 & 0.581 & 0.953 \\
\hline 16. & 284.45 & 983.771 & 0.642 & 0.953 \\
\hline 17. & 284.49 & 985.397 & 0.584 & 0.953 \\
\hline 18. & 284.27 & 995.447 & 0.713 & 0.953 \\
\hline 19. & 284.39 & 987.983 & 0.592 & 0.953 \\
\hline 20. & 284.31 & 1000.097 & 0.585 & 0.954 \\
\hline 21. & 284.64 & 989.293 & 0.652 & 0.953 \\
\hline 22. & 284.58 & 984.262 & 0.567 & 0.953 \\
\hline 23. & 284.69 & 984.713 & 0.634 & 0.953 \\
\hline 24. & 284.33 & 994.274 & 0.729 & 0.953 \\
\hline 25. & 284.61 & 984.035 & 0.734 & 0.953 \\
\hline 26. & 284.79 & 982.476 & 0.640 & 0.953 \\
\hline 27. & 284.81 & 986.261 & 0.565 & 0.953 \\
\hline 28. & 284.75 & 976.119 & 0.621 & 0.953 \\
\hline 29. & 285.86 & 970.877 & 0.376 & 0.956 \\
\hline 30. & 285.93 & 970.064 & 0.365 & 0.957 \\
\hline 31. & 284.86 & 968.894 & 0.684 & 0.953 \\
\hline 32. & 284.85 & 966.985 & 0.713 & 0.953 \\
\hline 33. & 284.83 & 973.492 & 0.658 & 0.953 \\
\hline 34. & 284.90 & 982.280 & 0.523 & 0.954 \\
\hline 35. & 284.64 & 975.753 & 0.709 & 0.953 \\
\hline 36. & 284.83 & 972.945 & 0.644 & 0.953 \\
\hline 37. & 284.62 & 982.768 & 0.599 & 0.953 \\
\hline 38. & 284.42 & 998.520 & 0.679 & 0.953 \\
\hline 39. & 284.35 & 997.152 & 0.628 & 0.953 \\
\hline 40. & 285.08 & 991.114 & 0.405 & 0.954 \\
\hline 41. & 285.03 & 986.623 & 0.479 & 0.954 \\
\hline 42. & 284.55 & 986.660 & 0.717 & 0.953 \\
\hline 43. & 284.64 & 996.952 & 0.466 & 0.954 \\
\hline
\end{tabular}


Table 2 Internal consistency of the items in the adapted Training Needs Analysis Questionnaire (Continued)

\begin{tabular}{|c|c|c|c|c|}
\hline Item number & $\begin{array}{l}\text { Scale mean if } \\
\text { item deleted }\end{array}$ & $\begin{array}{l}\text { Scale variance if } \\
\text { item deleted }\end{array}$ & $\begin{array}{l}\text { Corrected item-total } \\
\text { correlation }\end{array}$ & $\begin{array}{l}\text { Cronbach's alpha } \\
\text { if item deleted }\end{array}$ \\
\hline 44. & 284.69 & 992.197 & 0.506 & 0.954 \\
\hline 45. & 284.52 & 996.748 & 0.485 & 0.954 \\
\hline 46. & 284.47 & 989.722 & 0.607 & 0.953 \\
\hline 47. & 284.84 & 992.598 & 0.458 & 0.954 \\
\hline 48. & 284.91 & 986.496 & 0.455 & 0.954 \\
\hline 49. & 284.67 & 986.223 & 0.648 & 0.953 \\
\hline
\end{tabular}

(Table 3). Finally, examination of discriminant validity showed that the MSV was 0.49, which was below the AVE of 0.5. Discriminant validity was also determined by factor correlation matrix. The Pearson correlation coefficients between factors were $<0.7$, indicating the adapted TNAQ had discriminant validity. Detailed results are provided in Table 3.

\section{Construct validity}

The EFA produced three factors with eigenvalues of $>1.0$ and sampling adequacy of $85 \%$. Those factors were responsible for $36.77 \%$ of the variance. The rotation converged in 11 iterations. An examination of the KMO measure of sampling adequacy suggested that the sample was factorable $(\mathrm{KMO}=0.85)$. The Barlett's test of sphericity was significant $\left(\chi^{2}=\right.$ 4860.715, df 1176, $p<0.0001)$. Details for the factor loadings of TNAQ items are shown in Fig. 1.

As shown in Table 4, the TNAQ retained all 49 items and displayed a three-factor structure. Factor 2 included item number 13 (CEmONC activities) and was labeled CEmONC. Factor 3 included items 29 (surgical care) and 30 (anesthetic care) and was labeled intra-operative care. All other remaining items formed Factor 1, which was labeled general RMNH activities.

\section{CFA for TNAQ}

CFA was performed using generalized least squares estimation to compare the current and original 3-factor model of the scale. The model was found to be good $\left(\mathrm{x}^{2} / \mathrm{df}<3\right)$ and the CFI was optimal $(\mathrm{CFI}=1)$. However, the RMSEA was 0.185 indicating a poor model fit; this index was ignored because the other two indices showed a good fit and are commonly used and considered more predictive. The indices and related thresholds for interpretation are summarized in Table 5.

\section{Discussion}

This study aimed to translate and validate the Kiswahili version of TNAQ in RMNH services in Mwanza Region, Tanzania. Identification of training needs is an important step in developing effective and impactful capacity building interventions to bridge existing skill gaps among healthcare workers [23]. Rather than generating a new tool, which is time and resource intensive, we adapted and validated the Hennessy-Hicks TNAQ [19], which has been widely used in identifying training needs for a range of healthcare workers and is recognized by the WHO [19]. Adapting a TNA tool rather than constructing a new one is an approach used in other healthrelated specialties [32].

Our findings indicated that the adapted TNAQ had excellent reliability (internal consistency: Cronbach's alpha 0.954) [33], split-half reliability (Guttman split-half coefficient 0.84) [34], and inter-rater reliability (intraclass correlation coefficient 0.954) [21]. The convergent and discriminant validity of the scale were determined by the AVE, CR, and MSV. The AVE was 0.5, which was an acceptable value $(\leq 0.5)$ for convergent reliability, and the CR was 0.6, which indicated acceptable convergent reliability for an exploratory survey instrument. The MSV of 0.49 was less than the AVE value (0.5), and the correlations between factors was less than 0.7 , confirming the model had discriminant validity.

The internal consistency, split-half and intra-rater reliability of the TNAQ was determined. The TNAQ was

Table 3 Factor correlation matrix for the adapted Training Needs Analysis Questionnaire

\begin{tabular}{llll}
\hline Factor & General reproductive health & Intraoperative care & CEmONC \\
\hline General reproductive health & 1 & 0.310 & 0.431 \\
Intraoperative care & 0.310 & 1 & 0.431 \\
CEmONC & 0.431 & 0.407 & 1 \\
TNA total scores & 0.988 & 0.444 & 0.506 \\
\hline
\end{tabular}

CEmONC comprehensive emergency obstetric and newborn care

${ }^{\mathrm{a} C}$ Correlations significant at the 0.01 level (two-tailed) 


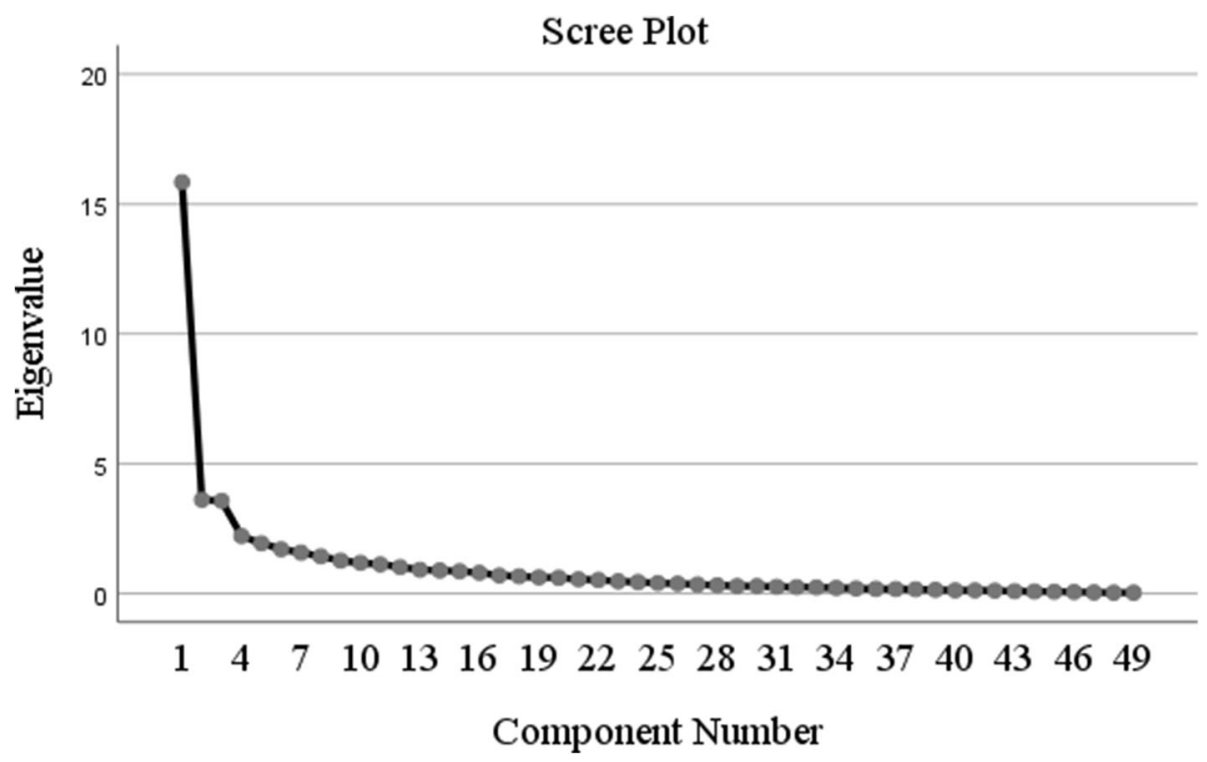

Fig. 1 Scree plot with eigenvalues to indicate the distribution of principal components

found with the internal consistency as measured by Cronbach's Alpha of 0.954 Indicating excellent reliability [33]. The Split-half reliability as measured by Guttman Split-half Coefficient was found to be 0.84 that demonstrates a very good level of internal consistency across sub-sets of the TNAQ. The Guttman Split-half Coefficient of 0.8 indicates a very good level and 0.6 to 0.7 represents the acceptable level however the values of higher than 0.95 may indicate redundancy of the tool therefore are not necessary [34]. The inter-rater reliability as determined by Intra-classes Correlation Coefficient of 0.954 was found, indicating excellent intra-rater reliability as it higher than the acceptable level of 0.7 [21]. Although the content validation procedure for the instrument in this study differed from a previous study that translated and validated the Hennessy-Hicks tool conducted in Greece [25], the overall internal consistency of 0.98 it that study was similar to our study. In addition, although the present study did not test for reproducibility, all reliability indices were within the acceptable threshold, indicating the adapted TNAQ was a consistent tool for assessing $\mathrm{RMNH}$ training needs in the Tanzanian context. It is important for a tool to have reliability to be acceptable, but it also needs to be valid [35, 36].

The TNAQ had acceptable face and content validity. We found a three-factor structure that reflected RMNH training needs. This differed from the five categories in the original TNAQ: (research/audit, communication/ teamwork, administrative/technical, management/supervisory, and clinical) [14]. This difference may be attributable to the focus of the tools; our adapted TNAQ focused on RMNH whereas the original TNAQ focused on general healthcare workers' training needs. The language and content of the translated questionnaire were clear and reflected Tanzanian culture. Most Tanzanians share key cultural aspects, such the Kiswahili language (spoken by about $95 \%$ of Tanzanians). Moreover, expression of culture and training needs may reflect those in other low-income countries with similar needs and cultural backgrounds [12]. Cross-cultural adaptation of an instrument is important in standardizing data that can be used in clinical, teaching, and policy analysis in the international arena. Numerous studies from different contexts and cultural backgrounds have reported similar psychometric properties for versions of the TNAQ [21, $25,37]$, indicating the TNAQ has acceptable criterion validity. Therefore, the findings of this study suggested that the TNAQ that considered the Tanzanian national guidelines on RMNH care and participants' culture (including Kiswahili) had an acceptable design and validity.

The convergent and discriminant validity were assessed by both calculated correlations and CFA. The EFA-derived structure was validated by CFA. The correlation coefficients and CFA indices were generally acceptable, indicating the model was suitable. A model is considered good if the $\mathrm{X}^{2} / \mathrm{df}=0.000$ is less that the recommended values of less than 3 . The chi-square statistic in our structured equation model with a statistically significant $\mathrm{p}$-value indicated the model did not fit the data adequately. Chi-square statistics are sensitive to sample size; therefore, given the small sample size used in this study, it indicates that there was insufficient power to detect differences between competing models [38]. However, use of the chi-square statistic has been debated, with some scholars suggesting chi-square tests are not 
Table 4 Principal component categorization indicating three factors from study participants $(n=153)$

\begin{tabular}{lll}
\hline SNO & Items & $\mathbf{1}$ \\
\hline 1 & Understanding gender equality issues relating to reproductive, maternal, child, and adolescent health & $\mathbf{3}$ \\
2 & $\begin{array}{l}\text { Delivering gender sensitive reproductive, maternal, child, and adolescent health services (e.g., providing } \\
\text { privacy for consultations, gender sensitive counselling approaches, involvement of men) }\end{array}$ & 0.557 \\
3 & Providing client/patient friendly reproductive, maternal, child, and adolescent health services & 0.505 \\
4 & Understanding and using maternal, newborn, and child health (MNCH) score cards [1] & 0.607 \\
5 & Providing focused antenatal care (FANC) according to WHO guidelines & 0.621 \\
6 & Offering malaria diagnosis with rapid diagnostic testing (RDT) & 0.652 \\
7 & Providing malaria treatment in pregnancy & 0.512 \\
8 & Providing education and counselling around voluntary counselling and testing (VCT) for HIV/AIDS & 0.557 \\
9 & Providing education, counselling, and support around HIV/AIDS prevention, care, and management for & 0.605 \\
adolescents & Competently managing uncomplicated deliveries & 0.491 \\
11 & Competently using the partograph for every woman in labor & 0.552 \\
12 & Competently providing basic emergency obstetric and newborn care (BEmONC) including: & 0.626
\end{tabular}

o Parenteral antibiotics

o Parenteral uterotonic drugs

o Parenteral anticonvulsants

o Manual removal of retained placenta

o Removal of retained products of conception

o Instrumental vaginal delivery

Basic neonatal resuscitation

13 Competently providing comprehensive emergency obstetric and newborn care (CEmONC)

o All BEmONC signal functions plus

o Surgery

o Blood transfusion

14 Competently managing severe intra- and postpartum hemorrhage

15 Responding effectively to women suffering from severe pre-eclampsia and eclampsia

16 Effectively resuscitating newborns using the newborn bag and mask (HBB-Helping Babies Breathe)

17 Identifying dangerous signs and complications in childbirth and effectively managing maternal and newborn referral for further investigations or treatment

18 Providing education and counselling on prevention of mother to child transmission of HIV (PMTCT)

20 Providing education, counselling, and support to mothers in early initiation of breast feeding (within $1 \mathrm{~h}$ of delivery) and exclusive breast feeding for 6 months Implementing the maternal, infant, and young child nutrition (MIYCN) program

22 Offering the Tanzania expanded program for immunization (EPI) 
Table 4 Principal component categorization indicating three factors from study participants $(n=153)$ (Continued)

\begin{tabular}{llc}
\hline SNO & Items & $\mathbf{1}$ \\
\hline 36 & Implementing effective disease surveillance and reporting & $\mathbf{3}$ \\
37 & Organizing your own time effectively & 0.639 \\
38 & Personally coping with change in the health service delivery & 0.623 \\
39 & Working as a member of a team & 0.721 \\
40 & Assuming a leadership role & 0.660 \\
41 & Developing leadership skills & 0.435 \\
42 & Mentoring and guiding other staff & 0.503 \\
43 & Supervision and management of community health workers & 0.758 \\
44 & Training of community health workers & 0.487 \\
45 & Undertaking effective data reporting and monitoring of service delivery & 0.514 \\
46 & Statistically analyzing your own data and using health facility data to understand local health challenges and inform & 0.500 \\
& service delivery & 0.635 \\
47 & Identifying research needs and designing locally relevant research & 0.470 \\
49 & Accessing research resources (e.g., time, money, information, equipment) & 0.450 \\
\hline
\end{tabular}

reliable because of sample size sensitivity, and instead encourage the use of multiple fit indices for a holistic view of the data [38-41] as they are less sensitive to the sample size [40]. Other studies suggested that the inferences based on p-values between large and small sample sizes may be the same but the chi-square values may differ significantly [38].

Our CFI value of 1 was higher than the recommended level (>0.95), but the RMSEA of 0.185 indicated that it was not a best fit (a smaller value indicates better model fit). The CFI does not give any useful information when RMSEA is more than 0.15 [42]. However, most indices were within the acceptable threshold indicating the instrument had excellent construct validity. Moreover, although there are several types of validity, construct validity is regarded the most important as it forms the basis of other types of validity and is scientifically viewed as the whole of validity [43]. We found the construct validity was acceptable, indicating that the adapted TNAQ was a valid instrument. This finding was similar to another study that found the tool had acceptable validity [25]. It was also similar to the original HennessyHicks instrument [14], which has been psychometrically tested for reliability and validity and adopted by the WHO [14, 19]. Therefore, the adapted TNAQ was scientifically validated to have potential to provide baseline data for identifying training needs among $\mathrm{RMNH}$ personnel in primary healthcare settings in Mwanza Region, Tanzania.

There were some limitations to this study that should be considered. First, this study was cross-sectional and only provided a snapshot in-time. The reliability and validity of competence (including behavior and attitudes) in the healthcare industry can better be measured on a continuum-over-time basis [44, 45]. Therefore, further studies that explore test-retest reliability and validity over time are warranted to validate the present findings. Second, there was a disproportionate number of items across the identified factors. Further studies could try to reduce the size of the instrument to keep it practical while maintaining the factor structure and its psychometric properties. Third, the sample size was relatively small and sufficient factor loading depends on the sample size. This limited the division of the dataset into subgroups; the item to participant ratio could therefore have been insufficient for factor loading. Moreover, the interpretation of the findings should take into consideration the inadequate sample size for providing robust estimates, especially for the latent variables included in the model and the participants' responses were clustered around the higher rank of

Table 5 Measures for confirmability factor analysis indicating model suitability

\begin{tabular}{lll}
\hline Indices & Measure observed & Threshold \\
\hline CFI & 1 & $>0.95=$ best, $>0.90=$ traditional, $>0.80=$ permissible \\
$X^{2}$ (CMIN/DF) & 0.000 & $<3=$ good, $<5=$ permissible \\
RMSEA & 0.185 & $<0.05=$ good, $0.05-0.10=$ moderate, $>0.10=$ poor \\
\hline
\end{tabular}


Likert scale. Finally, some participants did not respond to all items. Therefore, efforts should be made to recruit targeted participants that match the measured level of competence in further studies. However, this did not affect the validity and reliability of this study as missing data were excluded from the analysis.

\section{Conclusions}

The psychometric properties indicated that the Kiswahili translated and adapted TNAQ to be reliable and valid for identifying CPD needs for healthcare workers in the RMNH context at all levels of healthcare settings in Mwanza Region, Tanzania. The sufficient discriminative and evaluative psychometric properties provides evidence for further use of adapted, Kiswahili translated and validated TNAQ in RMNH in Tanzanian context. However, the applicability of the adapted TNAQ in the wider healthcare system remains unclear. Further studies with large sample sizes are required to test the use of the TNAQ in the wider healthcare system and learning opportunities.

\section{Abbreviations}

BEmONC: Basic emergency obstetric and newborn care; CEmONC: Comprehensive emergency obstetric and newborn care; CPD: Continuing professional development; TNA: Training needs analysis; IMPACT project: Improving access to reproductive, maternal and newborn health in Tanzania; TNAQ: Training Needs Analysis Questionnaire; RMNH: Reproductive, maternal, and newborn health; MMR: Maternal mortality rate; NMR: Neonatal mortality rate; WHO: World health organization; CFI: Comparative fit index; RMSEA: Root mean square error of approximation; CR: Composite reliability; AVE: Average variance extracted; EFA: Exploratory factor analysis; CFA: Comparative factor analysis

\section{Acknowledgements}

We would like to extend our thanks to Mwanza Regional Management including Regional Administrative Secretary, village executive officers, health management team, and participants for their support during the baseline survey.

\section{Authors' contributions}

TM drafted the manuscript and conducted data analysis. GE and KI reviewed the original TNAQ. TM, MM, JO, and DS conducted the data collection. DS, ES, MM, CM, LM, TM, Kl, and MT contributed to the conception and design of the study. TM, CM, KI, LM, EP GE, JO, MM, MM2, LS, SM, DS, ES, and MT provided critically important revisions of the manuscript. All authors read and approved the final manuscript.

\section{Funding}

This study received a grant from Global Affairs Canada through the Aga Khan Foundation Canada.

\section{Availability of data and materials}

The data that support the findings of this study are available from the Aga Khan University Monitoring and Evaluation Research Unit (MERL). There are some restrictions to the availability of these data due to license, so the data are not publicly available. However, the data may be made available from the authors upon reasonable request and with permission of the Aga Khan University MERL.

\section{Declarations}

\section{Ethics approval and consent to participate}

Approval to conduct this baseline survey was obtained from the National Institute for Medical Research in Tanzania (Registration Certificate: NIMR/
HQR/R.8a/Nol.IX/2517) after a review by the Aga Khan University Institutional Review Board in Tanzania. Permission to conduct the study was obtained from the Mwanza Regional Administrative Secretary. All participants provided oral and written informed consent after an explanation of the benefit, potential harm, duration of the interview, and the right to refuse or withdrawal from the study at any point if they wished to do so.

\section{Consent for publication}

Not applicable.

\section{Competing interests}

Authors declare that they have no competing interests.

\section{Author details}

'School of Nursing and Midwifery, the Aga Khan University, Dar es Salaam, Tanzania. ${ }^{2}$ School of Nursing and Midwifery, the Aga Khan University, Kampala, Uganda. ${ }^{3}$ Department of Population Health, the Aga Khan University, Nairobi, Kenya. ${ }^{4}$ Aga Khan Health Services, Mwanza, Tanzania. ${ }^{5}$ Community Development, Ministry of Health, Gender, Elderly and Children, Dodoma, Tanzania. ${ }^{6}$ Regional Reproductive and Child Health Coordinator, Mwanza, Tanzania. ${ }^{7}$ Aga Khan Foundation, Dar es Salaam, Tanzania.

Received: 24 April 2020 Accepted: 23 June 2021

Published online: 24 July 2021

\section{References}

1. WHO. WHO, UNICEF, UNFPA and The World Bank estimates. Trends in maternal mortality: 1990 to 2013. 2013.

2. TDHS. Tanzania Demographic Health Survey (TDHS) 2010 and 2015/ 16. 2016.

3. Kruk ME, Leslie HH, Verguet S, Mbaruku GM, Adanu RMK, Langer A. Quality of basic maternal care functions in health facilities of five African countries: an analysis of national health system surveys. Lancet Glob Health. 2016: 4(11):e845-55

4. Frenk J, Chen L, Bhutta ZA, Cohen J, Crisp N, Evans T, et al. Health professionals for a new century: transforming education to strengthen health systems in an interdependent world. Rev Peru Med Exp Salud Publica. 2011;28(2):337-41.

5. Campbell OM, Calvert C, Testa A, Strehlow M, Benova L, Keyes E, et al. The scale, scope, coverage, and capability of childbirth care. Lancet. 2016; 388(10056):2193-208.

6. Lenters L, Wazny K, Bhutta ZA. Management of severe and moderate acute malnutrition in children. In: Black RE, Laxminarayan $\mathrm{R}$, Temmerman $\mathrm{M}$, Walker $\mathrm{N}$, editors. Reproductive, maternal, newborn, and child health: disease control priorities. vol. 2. 3rd Ed. Washington (DC): The International Bank for Reconstruction and Development/The World Bank; 2016.

7. Bhutta ZA, Ali S, Cousens S, Ali TM, Haider BA, Rizvi A, et al. Alma-Ata: rebirth and revision 6 interventions to address maternal, newborn, and child survival: what difference can integrated primary health care strategies make? Lancet. 2008;372(9642):972-89.

8. Frenk J, Chen L, Bhutta ZA, Cohen J, Crisp N, Evans T, et al. Health professionals for a new century: transforming education to strengthen health systems in an interdependent world. Lancet. 2010;376(9756):1923-58.

9. Ona S, Easter SR, Prabhu M, Wilkie G, Tuomala RE, Riley LE, et al. Diagnostic validity of the proposed Eunice Kennedy Shriver National Institute of Child Health and Human Development Criteria for Intrauterine Inflammation or Infection. Obstet Gynecol. 2019;133(1):33-9.

10. Ariff S, Soofi SB, Sadiq K, Feroze AB, Khan S, Jafarey SN, et al. Evaluation of health workforce competence in maternal and neonatal issues in public health sector of Pakistan: an Assessment of their training needs. BMC Health Serv Res. 2010;10:319.

11. Trevino-Siller S, Gonzalez-Hernandez D, Fritz J, Olvera Garcia M, Montoya A, Sanchez-Dominguez $M$, et al. Is it possible to incorporate evidence-based professional midwifery practices into public health services in Mexico? Women Birth. 2019;33(3):240-50.

12. Bailey C, Blake C, Schriver M, Cubaka VK, Thomas T, Martin Hilber A. A systematic review of supportive supervision as a strategy to improve primary healthcare services in Sub-Saharan Africa. Int J Gynaecol Obstet. 2016;132(1):117-25.

13. Lorwald AC, Lahner FM, Nouns ZM, Berendonk C, Norcini J, Greif R, et al. The educational impact of Mini-Clinical Evaluation Exercise (Mini-CEX) and 
Direct Observation of Procedural Skills (DOPS) and its association with implementation: a systematic review and meta-analysis. PLoS One. 2018; 13(6):e0198009.

14. Hicks C, Hennessy D, Barwell F. Development of a psychometrically valid training needs analysis instrument for use with primary health care teams. Health Serv Manage Res. 1996;9(4):262-72.

15. Hennessy D, Hicks C, Koesno H. The training and development needs of midwives in Indonesia: paper 2 of 3. Hum Resour Health. 2006;4:9.

16. Hicks $C$, Tyler C. Assessing the skills for family planning nurse prescribing: development of a psychometrically sound training needs analysis instrument. J Adv Nurs. 2002;37(6):518-31.

17. Orwa J, Mantel M, Mugerwa M, Brownie S, Pallangyo ES, Mwasha L, et al. Maternal healthcare services use in Mwanza Region, Tanzania: a crosssectional baseline survey. BMC Pregnancy Childbirth. 2019;19(1):474.

18. Anthoine E, Moret L, Regnault A, Sebille V, Hardouin JB. Sample size used to validate a scale: a review of publications on newly-developed patient reported outcomes measures. Health Qual Life Outcomes. 2014;12:176.

19. Hicks C, Hennessy D. The use of a customized training needs analysis tool for nurse practitioner development. J Adv Nurs. 1997;26(2):389-98.

20. Wangensteen S, Finnbakk E, Adolfsson A, Kristjansdottir G, Roodbol P, Ward $\mathrm{H}$, et al. Postgraduate nurses' self-assessment of clinical competence and need for further training. A European cross-sectional survey. Nurse Educ Today. 2018;62:101-6.

21. Pinxten WJL, Fitriana E, De Jong C, Klimas J, Tobin H, Barry T, et al. Excellent reliability and validity of the Addiction Medicine Training Need Assessment Scale across four countries. J Subst Abuse Treat. 2019;99:61-6.

22. Shewade HD, Jeyashree K, Kalaiselvi S, Palanivel C, Panigrahi KC. Assessment of community-based training of medical undergraduates: development and validation of a competency-based questionnaire. Educ Health (Abingdon). 2016;29(3):244-9.

23. Tahir M, Kadir K, Apipi M, Ismail SM, Yusof ZYM, Yap AU. Translation and adaptation of the diagnostic criteria for temporomandibular disorders into the Malay language: psychometric evaluation of contents. J Oral Facial Pain Headache. 2020:34(4):323-30.

24. Mehta S, Bastero-Caballero RF, Sun Y, Zhu R, Murphy DK, Hardas B, et al. Performance of intraclass correlation coefficient (ICC) as a reliability index under various distributions in scale reliability studies. Stat Med. 2018;37(18):2734-52.

25. Markaki A, Antonakis N, Hicks CM, Lionis C. Translating and validating a Training Needs Assessment tool into Greek. BMC Health Serv Res. 2007;7:65.

26. Gutteridge TP, Lang CP, Turner AM, Jacobs BW, Laurens KR. Criterion validity of the Psychotic-Like Experiences Questionnaire for Children (PLEQ-C). Schizophr Res. 2020;220:78-84.

27. Marsh HW, Guo J, Dicke T, Parker PD, Craven RG. Confirmatory Factor Analysis (CFA), Exploratory Structural Equation Modeling (ESEM), and SetESEM: optimal balance between goodness of fit and parsimony. Multivariate Behav Res. 2019;55(1):102-19.

28. Sass DA, Schmitt TA. A comparative investigation of rotation criteria within exploratory factor analysis. Multivariate Behav Res. 2010;45(1):73-103.

29. Barnett SD, Sarin EL, Henry L, Halpin L, Pritchard G, Speir AM. Confirmatory factor analysis of the Minnesota living with heart failure questionnaire among patients following open heart surgery for valve dysfunction. Quality of life research: an international journal of quality of life aspects of treatment care rehabilitation. 2019;28(1):267-75.

30. Pierce KM, Maxwell SD, Olino TM, Cooper S, Ellman LM. Factor structure, convergent, and divergent validity of the prodromal questionnaire-negative symptom subscale. Assessment. 2021;28(1):153-68.

31. Hair JF. Multivariate data analysis: a global perspective 7. Upper Saddle River: Prentice Hall; 2009.

32. Gaspard J, Yang CM. Training needs assessment of health care professionals in a developing country: the example of Saint Lucia. BMC Med Educ. 2016; 16:112.

33. Kuhlmann AYR, Lahdo N, Staals LM, van Dijk M. What are the validity and reliability of the modified Yale Preoperative Anxiety Scale-Short Form in children less than 2 years old? Paediatr Anaesth. 2019;29(2):137-43.

34. Luna FG, Roca J, Martin-Arevalo E, Lupianez J. Measuring attention and vigilance in the laboratory vs. online: the split-half reliability of the ANTI-Vea. Behav Res Methods. 2021;53(3):1124-47.

35. Gronlund CF, Soderberg A, Dahlqvist V, Andersson L, Isaksson U. Development, validity and reliability testing the Swedish Ethical Climate Questionnaire. Nurs Ethics. 2019:26(7-8):2482-93.
36. DeVon HA, Block ME, Moyle-Wright P, Ernst DM, Hayden SJ, Lazzara DJ, et al. A psychometric toolbox for testing validity and reliability. J Nurs Scholarsh. 2007;39(2):155-64

37. Kilbertus S, Pardhan K, Zaheer J, Bandiera G. Transition to practice: evaluating the need for formal training in supervision and assessment among senior emergency medicine residents and new to practice emergency physicians. CJEM. 2019;21(3):418-26.

38. Bergh D. Chi-squared test of fit and sample size - a comparison between a random sample approach and a chi-square value adjustment method. J Appl Meas. 2015;16(2):204-17.

39. Lin S. Sample size requirement for detecting interference under the chisquare model. Hum Hered. 2001;52(4):201-9.

40. Tehranchi A, Neshat Doost HT, Amiri S, Power MJ. The role of character strengths in depression: a structural equation model. Front Psychol. 2018;9: 1609.

41. Xia Y, Yang Y. The Influence of Number of Categories and Threshold Values on Fit Indices in Structural Equation Modeling with Ordered Categorical Data. Multivariate Behav Res. 2018;53(5):731-55.

42. Savalei V. On the Computation of the RMSEA and CFI from the Mean-AndVariance Corrected Test Statistic with Nonnormal Data in SEM. Multivariate Behav Res. 2018;53(3):419-29.

43. Strauss ME, Smith GT. Construct validity: advances in theory and methodology. Annu Rev Clin Psychol. 2009;5:1-25

44. Franklin B, Gasco J, Uribe T, VonRitschl RH, Hauck E. Diagnostic accuracy and inter-rater reliability of 64-multislice 3D-CTA compared to intra-arterial DSA for intracranial aneurysms. J Clin Neurosci. 2010;17(5):579-83.

45. Sam AH, Field SM, Collares CF, van der Vleuten CPM, Wass VJ, Melville C, et al. Very-short-answer questions: reliability, discrimination and acceptability. Med Educ. 2018;52(4):447-55.

\section{Publisher's Note}

Springer Nature remains neutral with regard to jurisdictional claims in published maps and institutional affiliations.

\section{Ready to submit your research? Choose BMC and benefit from:}

- fast, convenient online submission

- thorough peer review by experienced researchers in your field

- rapid publication on acceptance

- support for research data, including large and complex data types

- gold Open Access which fosters wider collaboration and increased citations

- maximum visibility for your research: over $100 \mathrm{M}$ website views per year

At $\mathrm{BMC}$, research is always in progress.

Learn more biomedcentral.com/submission 